\title{
Identification of the Ambient Response Relationship in Neutron Counting and Scintillation Measurement Systems
}

\author{
Jackson Nicholas Wagner ${ }^{*}$, Craig Marianno \\ Department of Nuclear Engineering, Texas A\&M University, Texas, United States of America
}

Email address:

jackwags@tamu.edu (J. N. Wagner), marianno@tamu.edu (C. Marianno)

${ }^{*}$ Corresponding author

\section{To cite this article:}

Jackson Nicholas Wagner, Craig Marianno. Identification of the Ambient Response Relationship in Neutron Counting and Scintillation Measurement Systems. Radiation Science and Technology. Vol. 7, No. 1, 2021, pp. 7-14. doi: 10.11648/j.rst.20210701.12

Received: February 19, 2021; Accepted: March 2, 2021; Published: March 12, 2021

\begin{abstract}
Radiation detection for nuclear security frequently employs neutron counting and scintillation systems simultaneously. One potential issue, particularly when searching a large area, is understanding the ambient (or background) response of these systems throughout the operation. This is easily mitigated for the scintillation system but remains a problem for neutron counting systems. Operational data and previous research have shown that a correlation appears between the neutron count rate and the count rate at high energies in the scintillation system (energies greater than $4 \mathrm{MeV}$ ) in background conditions. To understand the cause of the correlation, background measurements were performed using sodium iodide (NaI) and polyvinyl toluene (PVT) scintillation systems. These detectors were calibrated to high energy scales such that their spectra would show energies up to $70 \mathrm{MeV}$ and $85 \mathrm{MeV}$, respectively. Results show that at least one statistical mode appeared in the spectra on these energy scales (particularly between $5 \mathrm{MeV}$ and $60 \mathrm{MeV}$ ). The energy and maximum probability of these modes varied with orientation, and they were dependent upon the detector thickness with respect to the vertical axis and the detector area perpendicular to that axis, respectively. The modes' energies also matched the expected energy deposition from background muons in the detectors with path lengths equal to one of the detectors' dimensions. These data matched results from simulations of background muons interacting with these detectors calculated using MCNP, and they similarly matched muon energy spectra calculated from possible path lengths through the detectors using Python. These results indicate that scintillation measurements at energies higher than those employed in typical nuclear security operations are the result of background muons. Since these muons are produced similar processes as background neutrons, the count rate of these particles could potentially be applied to better characterize the background in neutron counting systems.
\end{abstract}

Keywords: Muon Detection, Background Radiation, Nuclear Security

\section{Introduction}

The threat of nuclear terrorism is a concern for many nations. A nuclear terrorist attack would most likely occur at a public event and would use a radiological dispersal device (RDD - commonly known as a "dirty bomb") or an improvised nuclear device (IND). A common tool for securing these events is the mobile search system. This employs gamma spectroscopy and neutron counting techniques during a large-area search to locate radioactive material.

To efficiently locate sources of radiation, the mobile search system detectors must have well understood ambient (or "background") responses. This is simple for a gamma spectroscopy system as the background has a spectrum that is unique from any illicit gamma ray-emitting material $[1,2]$. The system also has the benefit that the source of most background gamma rays is the Earth, which produces little variance in the overall count rate during a mobile search. However, the background response in a neutron counting system is much more difficult to characterize because the user is only provided a count rate. This is compounded by the fact that the main source of background neutrons is secondary cosmic ray interactions in the Earth's upper atmosphere (mainly through spallation processes) [3]. This and the physics of neutron interactions means the neutron count rate can vary with elevation changes along with the presence and composition of surrounding buildings or 
material. This makes it more difficult to determine if a change in neutron count rate is due to one of these background changes or from an illicit source.

Previous work has suggested that employing a different data stream from the gamma spectrometer has the potential to improve the neutron background's characterization during mobile searches. Specifically, that the background count rate of high energy signals in the gamma spectrometer (greater than $3 \mathrm{MeV}$ ) is correlated with the background neutron count rate. This was initially indicated by operational data, and its presence has been shown with varying concrete shielding from parking garages [4]. In a continuing effort to explain and generalize this correlation, this work sought to explain the cause of these high energy signals.

\section{Materials and Methods}

To determine the source of high energy signals produced in gamma spectrometers, spectra from long background measurements were compared to simulations of candidate particles interacting with the same detectors. Measurements were performed with spectrometers commonly used during mobile searches: sodium iodide (NaI) and polyvinyl toluene (PVT) scintillator detectors. The NaI detector had dimensions of 2"-by-4"-by-16" (5.08 cm-by-10.16 cm-by-40.64 cm) and was manufactured by Alpha Spectra Inc. (model \#: 8D16X64A5/3.5), and the PVT detector had dimensions of 2.5"-by-6.5"-by-24.5" (6.35 cm-by-16.51 cm-by-62.23 cm) and was manufactured by Alpha Spectra Inc. (model \#: P8124X96/2) [5- 7].
Signals from these detectors were collected using an Ortec digiBASE. This multi-channel analyzer (MCA) was set to 1024 channels and was calibrated so particles over $10 \mathrm{MeV}$ could be registered [8]. The scale of the NaI spectrum was calibrated to approximately $70 \mathrm{MeV}$ using the photopeaks from ${ }^{137} \mathrm{Cs}$ and ${ }^{22} \mathrm{Na}$, while the PVT scale was set to approximately $85 \mathrm{MeV}$ using the Compton Edge energies for the $662 \mathrm{keV}$ photon from ${ }^{137} \mathrm{Cs}$ and the $1274 \mathrm{keV}$ photon from ${ }^{22} \mathrm{Na}$. The spectral features aligning with the respective channels are shown in Table 1 . The detectors were connected to a computer running Ortec's MAESTRO software for Windows as shown in the block diagram in Figure 1 [9]. To minimize the variance in the measured spectra, data were collected using this system for 36 hours for each measurement scenario.

The potential particles of interest in these detectors as calibrated are high energy gamma rays, protons, and muons (negatively charged particles 200 times more massive than electrons). All three of these particles are the result of secondary cosmic ray interactions in the atmosphere. In addition to the gamma rays, the protons and muons interact with the NaI and PVT detectors [1]. Research has shown that these detectors have a linear response as a function of energy for protons and an increasingly non-linear response as particle mass increases [10, 11]. Previous measurements have also shown that muons produce a peak (or mode) in a scintillator's spectrum at energy scales similar to those described above. However, these experiments were performed using coincidence measurements and mainly for laboratory applications [12].

Table 1. Calibration information for NaI and PVT detectors.

\begin{tabular}{|c|c|c|c|c|c|c|}
\hline \multirow{3}{*}{ Detector } & \multicolumn{6}{|c|}{ Isotope } \\
\hline & & & Units & ${ }^{137} \mathrm{Cs}$ & ${ }^{22} \mathrm{Na}$ & \\
\hline & Gamma Ray Energy & & {$[\mathrm{keV}]$} & 662 & 511 & 1274 \\
\hline \multirow{4}{*}{$\mathrm{NaI}$} & \multirow{4}{*}{$\begin{array}{l}\text { Photopeak } \\
\text { digiBASE Voltage } \\
\text { Calibration }\end{array}$} & \multirow{4}{*}{$\begin{array}{l}\text { Energy } \\
\text { Channel }\end{array}$} & {$[\mathrm{keV}]$} & 662 & 511 & 1274 \\
\hline & & & & $10.5(1.0)$ & $8(1)$ & $19.5(1.0)$ \\
\hline & & & {$[\mathrm{V}]$} & 500 & & \\
\hline & & & {$\left[\mathrm{keV} \mathrm{ch}{ }^{-1}\right]$} & $68.0(10.7)$ & & \\
\hline \multirow{4}{*}{ PVT } & \multirow{4}{*}{$\begin{array}{l}\text { Compton Edge } \\
\text { digiBASE Voltage } \\
\text { Calibration }\end{array}$} & \multirow{4}{*}{$\begin{array}{l}\text { Energy } \\
\text { Channel }\end{array}$} & {$[\mathrm{keV}]$} & 477.65 & - & 1061.18 \\
\hline & & & & $8(1)$ & - & $15(1)$ \\
\hline & & & {$[\mathrm{V}]$} & 720 & & \\
\hline & & & {$\left[\mathrm{keV} \mathrm{ch}{ }^{-1}\right]$} & $83.3(16.8)$ & & \\
\hline
\end{tabular}

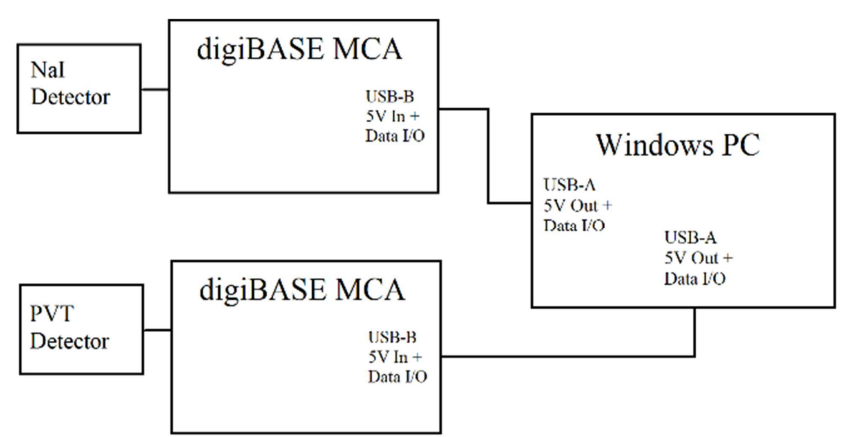

Figure 1. Block diagram of connections of detectors to computer for all measurements.

Considering the literature more broadly suggests that the muons are the most likely candidate of these three particles. Previous work has shown that the proton flux at the Earth's surface is two orders of magnitude smaller than that of muons [13]. Published research on the photon component of cosmic rays at the Earth's surface suggests their flux is negligible compared to muons [3, 14]. The low flux for protons and gamma rays (relative to muons) would make it difficult to distinguish their respective spectral features from those of muons. Additionally, the lack of literature on the characteristics of high-energy gamma rays near Earth's surface would make their simulation difficult because of the inability to accurately model the particles' source [3, 14].

As stated above, these muons are the product of cosmic ray interactions. The primary cosmic rays are mainly comprised of protons from outer space with energies much greater than 1 
$\mathrm{GeV}$. When these particles interact with the nitrogen and oxygen atoms of the atmosphere, strong nuclear processes produce a shower of exotic particles, initially comprised of short-lived kaons and pions (mean lifetimes: 12-50 ns). These eventually decay into muons, and many have enough kinetic energy to reach the Earth's surface before decaying (mean lifetime: $2.2 \mu \mathrm{s}$ ) [3].

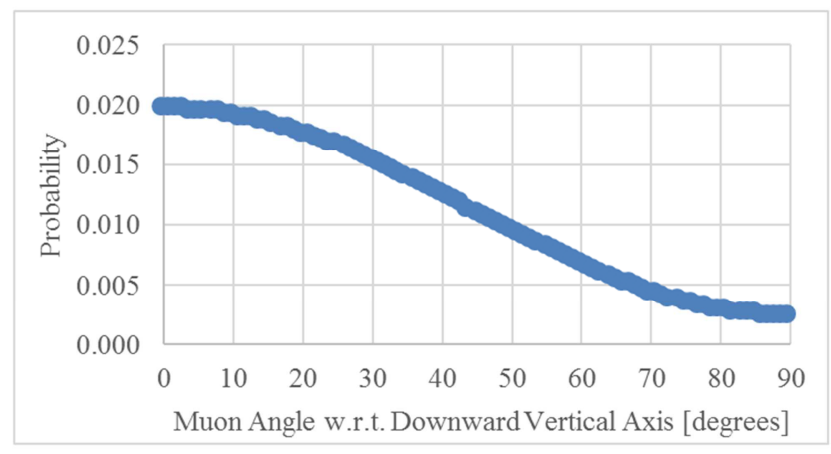

Figure 2. Muon direction probability density function [15].

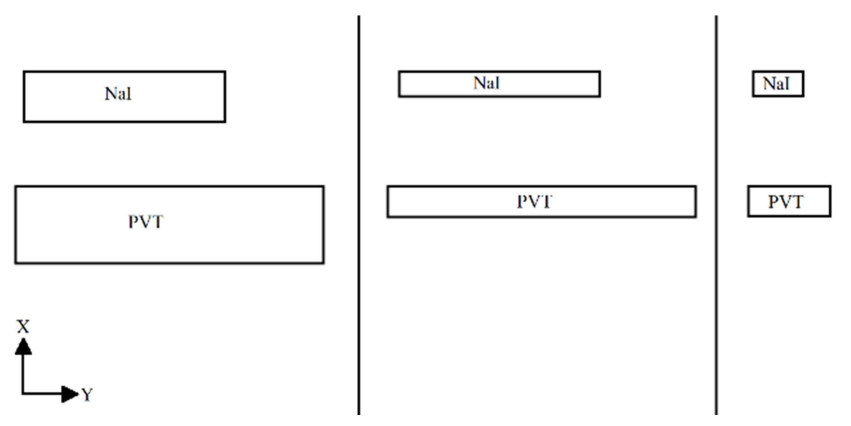

Figure 3. Diagrams showing the top-down views of the NaI and PVT detectors for the orientations used, which were named "Horizontal, Long Side Up" (left), "Horizontal, Short Side Up" (center), and "Vertical" (right). Each detector geometry is shown on the same relative scale.

Since the muons are charged particles, detector orientation was an important parameter for this research. Energy deposition of the charged particle depends on its path length through the detector, and these cosmic muons have a mostly downward trajectory as indicated in Figure 2. This figure illustrates that $40 \%$ of cosmic muons have an angle of incidence less than 20 degrees from the vertical axis and $90 \%$ have an angle less than 62 degrees [15]. This characteristic would likely produce variance in the measured spectra with different detector orientations, particularly because changing the detector geometry relative to the vertical axis will change the distribution of possible muon paths, thus changing the energy spectrum. Three separate detector orientations were used to collect data. For the purposes of this analysis, the orientations are referred as "Vertical," where the smallest detector face was parallel to the ground; "Horizontal, Short Side Up," where the second largest detector face was parallel; and "Horizontal, Long Side Up," where the largest detector face was parallel. These orientations are illustrated in Figure 3.

Because these particles interact with the detectors differently than gamma rays, the measured spectra required a slightly different terminology for analysis than gamma ray spectra.
Specifically, analysis of the spectra looked for global and local modes in a statistical sense (i.e. the most frequent value of a spectrum or a subsection of it). This is similar to that for a gamma ray spectrum, but those modes are referred as "photopeaks" (or simply "peaks") because they correlate with gamma rays undergoing a photoelectric process within the detector. Since muons and protons cannot undergo a photoelectric process, it would be incorrect to refer to statistical modes of a spectrum as "peaks." Therefore, analysis of measured and calculated spectra will refer to the modal data.

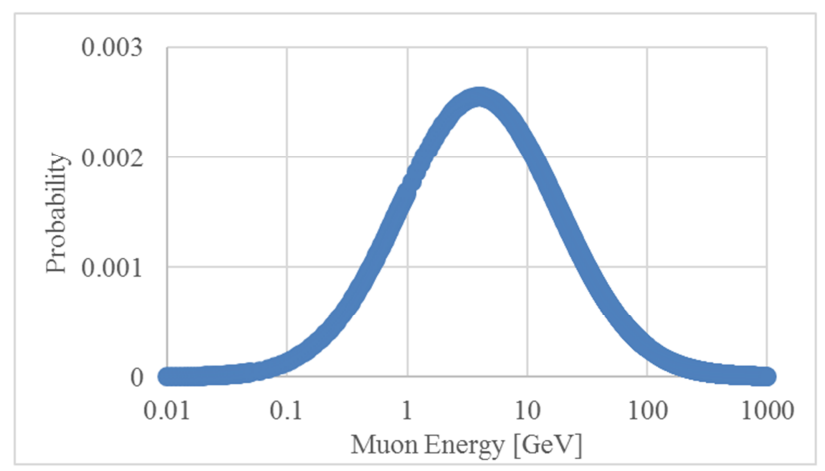

Figure 4. Muon energy probability density function used for MCNP simulations, which was modeled as a log-normal distribution with a mean of $4 \mathrm{GeV}$ and a standard deviation of $220 \mathrm{GeV}$ to match literature [15].

If the particles are muons, an approximation of the expected energy spectrum can be calculated for each detector in each orientation. This was done by calculating the distribution of potential path lengths through the detector using a Python script. For all possible muon entry points on the detector's surface, this script calculated the possible path lengths a muon could travel from that entry point through the detector for all possible directions. These directions fell on cones whose half angles were governed by the distribution of angles with the vertical axis shown in Figure 2. This script had a $2 \mathrm{~mm}$ resolution of entry locations, and a 1 degree resolution of both polar angles for direction, where these resolutions were limited by available computer memory. The energy spectrum was approximated by multiplying these path lengths by a muon's minimum linear energy deposition. This value is 4.785 $\mathrm{MeV} \mathrm{cm}{ }^{-1}$ for $\mathrm{NaI}$ and $2.019 \mathrm{MeV} \mathrm{cm}^{-1}$ for PVT. The actual linear energy deposition does depend on energy, but the minimum values for each material appear near the mean energy of a background muon, which is $4 \mathrm{GeV}$ on a log-normal distribution $[15,16]$. These energies were then collected in $50 \mathrm{keV}$ bins to produce an energy spectrum to be compared against measured data.

To confirm that the particles measured are muons, the recorded spectra were also compared against MCNP simulation results [17]. These computer models simulated the muon's response in the two scintillators in the orientations used in the physical measurements. The cosmic muon source was modeled with a direction-biased spectrum derived from cited measurements as shown in Figure 2. The muon's energy spectrum was modeled as a log-normal distribution with a mean of $4 \mathrm{GeV}$ and standard deviation of $220 \mathrm{GeV}$, where the 
latter was selected to match the shape of the spectrum in literature as shown in Figure 4 [15]. The simulation used a disk for the muon source placed $10 \mathrm{~m}$ above a simplified detector geometry. Biasing was applied to source the muons' direction as a function of radius to improve the speed of the simulation's convergence. In addition, the F8 tally had a Gaussian energy broadening modifier to simulate the detector's energy variance.

\section{Results and Discussion}

The measurements described above produced the spectra shown in Figure 5 for $\mathrm{NaI}$ and those for PVT are shown in Figure 6 . These spectra show distinct modes that vary with the orientation of the detector. Data about these modes are shown in Table 2. In NaI, modes appeared at $25.5 \pm 4.0 \mathrm{MeV}$ in the Horizontal-Long Side Up orientation, at $28.9 \pm 4.5 \mathrm{MeV}$ and 48.0 \pm 7.5 MeV in the Horizontal-Short Side Up orientation, and at $27.1 \pm 4.3 \mathrm{MeV}$ in the Vertical orientation. The count rates of all signals above $8.0 \mathrm{MeV}$ were $8.50 \pm 0.01 \mathrm{~s}^{-1}$, $6.51 \pm 0.01 \mathrm{~s}^{-1}$, and $4.94 \pm 0.01 \mathrm{~s}^{-1}$, respectively. In PVT, modes appeared at $9.6 \pm 1.9 \mathrm{MeV}$ in the Horizontal-Long Side Up orientation, at $11.8 \pm 2.4 \mathrm{MeV}$ and $26.1 \pm 5.3 \mathrm{MeV}$ in the Horizontal-Short Side Up orientation, and at $11.3 \pm 2.3 \mathrm{MeV}$ in the Vertical orientation. The count rates of all signals above $4.8 \mathrm{MeV}$ were $15.69 \pm 0.01 \mathrm{~s}^{-1}, 10.88 \pm 0.01 \mathrm{~s}^{-1}$, and $10.02 \pm 0.01$ $\mathrm{s}^{-1}$, respectively.

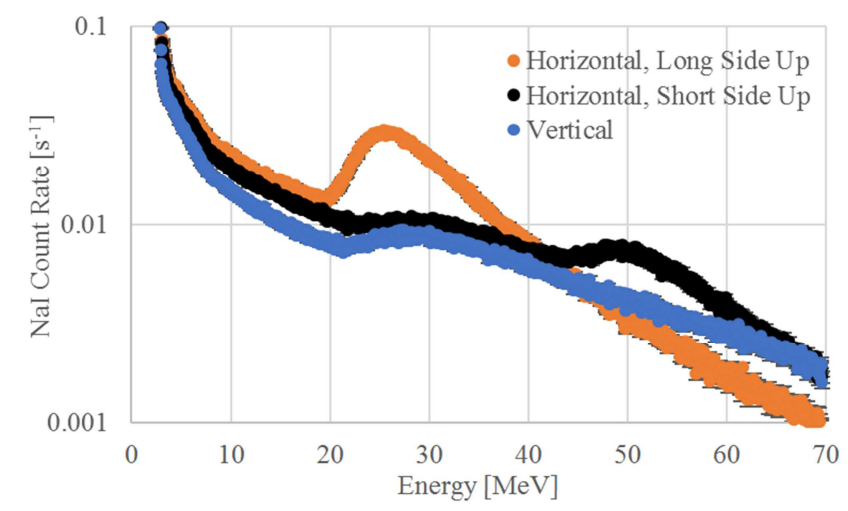

Figure 5. Measured energy spectra for NaI detector in the Horizontal-Long Side Up (orange), Horizontal- Short Side Up (black), and Vertical (blue) orientations. 1-sigma error bars are included but most are obscured by the data markers.

These data show that the count rates vary with the detector's geometry, following a positive relationship with the detector's surface area orthogonal to the vertical axis. This indicates that the detected particles are produced at some distance either above or below the detectors. That aspect fits with the expectation that the particles are secondary cosmic rays produced in the atmosphere. The variance in the spectra with differing orientation suggest that the particles deposit energy as a function of path length and thus have electric charge. Specifically, the spectral features suggest that the charged particles are muons. This is indicated in the data for the Horizontal, Long Side Up orientations, whose modes' energies are statistically the same as the expected muon energy deposition for the detectors' two smaller dimensions as shown in Table 3.

For comparison against these measured spectra, the plots produced from the Python calculations of muon spectra are shown in Figure 7 for $\mathrm{NaI}$ and Figure 8 for PVT. The general shape of the calculated spectra match those from measurements, particularly where at least one mode is visible in each spectrum and instances where a second modes distinctly appear in some orientations. Similar to the measured data described above, the energy of a given mode correlates to a muon path length equal to one of the detector's dimensions. Both the modes' locations and the spectra's overall similarity indicate further that the measured spectra are produced by muons. With this result, it is worthwhile to consider the physics that contribute to the shape and variance of the spectra.

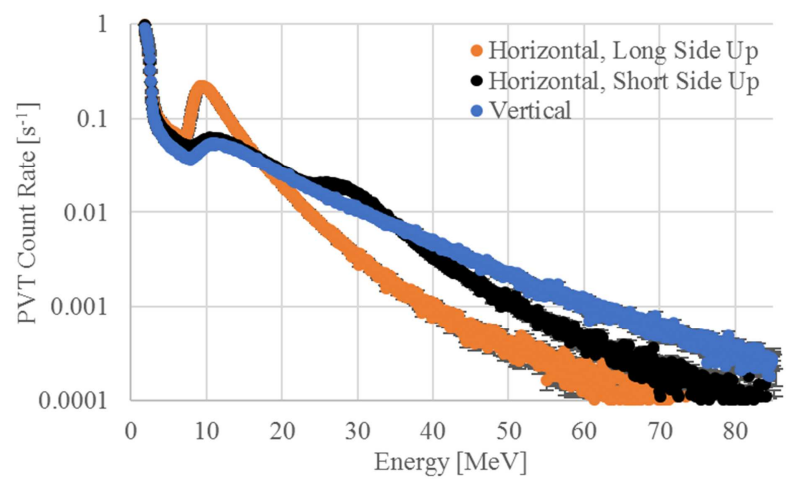

Figure 6. Measured energy spectra for PVT detector in the Horizontal-Long Side Up (orange), Horizontal-Short Side Up (black), and Vertical (blue) orientations. 1-sigma error bars are included but most are obscured by the data markers.

To better analyze the physics that cause these spectra, Figure 9 shows a magnified version of the calculated spectra for PVT in the Horizontal, Short Side Up orientation labeled for different spectral features. The idealized nature of this calculated spectrum aids in the precision of describing the physics. First are the modes of the spectrum, labelled B and D. The mode at $\mathrm{B}$ appears at $12.8 \mathrm{MeV}$ indicates a path length of $6.35 \mathrm{~cm}$, the detector's shortest dimension. The mode at D appears at $33.3 \mathrm{MeV}$ similarly indicates a path length of 16.51 $\mathrm{cm}$, the detector's middle dimension. Considering that with the detector's orientation indicate that $\mathrm{B}$ is the result of muons entering one of the detector's vertical faces (i.e. two of its larger "sides") with an approximately horizontal trajectory, and D is the result of muons entering the detector's upper horizontal face (i.e. its "top") with an approximately vertical trajectory. Diagrams of these muon paths can be seen in Figures 10 (b) and 10 (d), respectively. In this orientation, both modes are equally likely with probabilities of 0.032 and 0.035 , respectively, and this is the result of the distribution of the muon trajectories and the detector's geometry. Muons are 8.4 times more likely to appear travelling vertically than horizontally (see Figure 2), but the detector's vertical face is 2.6 times larger than its horizontal face, which means there are 5.2 times more potential horizontal muon paths than vertical ones. This suggests that the peak 
probability of mode D would be 1.6 times that of mode B. While Figure 9 shows that the probability of $\mathrm{D}$ is only 1.1 times that of $\mathrm{B}$, the difference from this expectation is likely a result of the binning scheme for the calculations.

Table 2. Modal data for NaI and PVT measurements by orientation including modes'full-widths at half maximum (FWHMs), when its calculation was possible. Missing data for "Mode 2" indicate that only one mode existed for a given measurement.

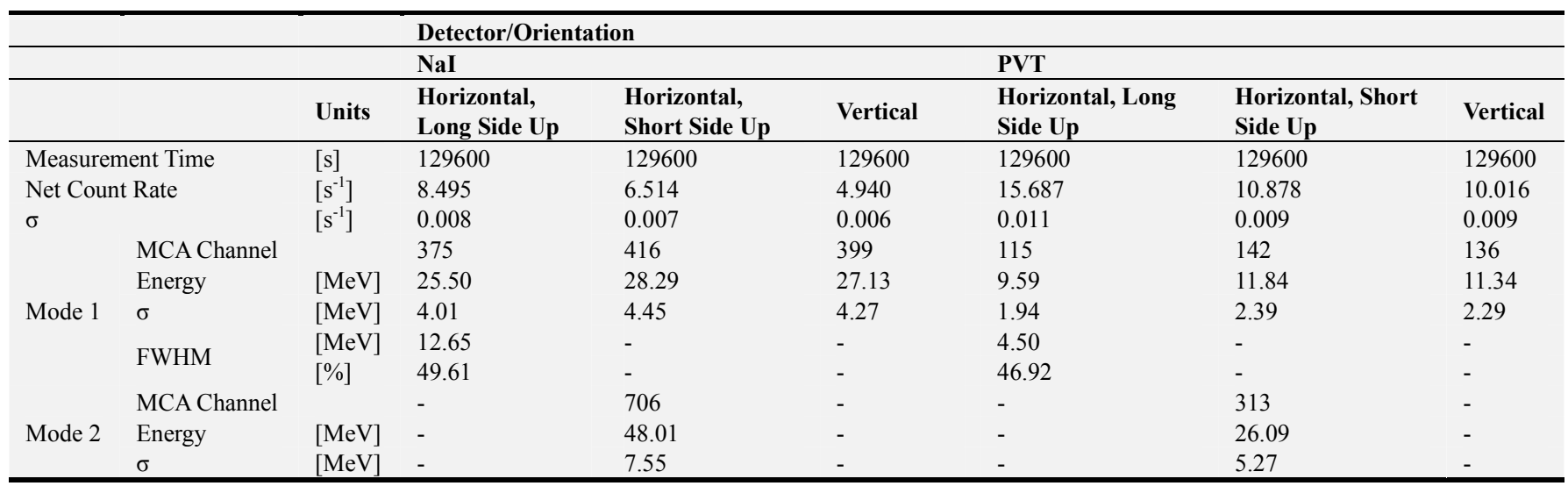

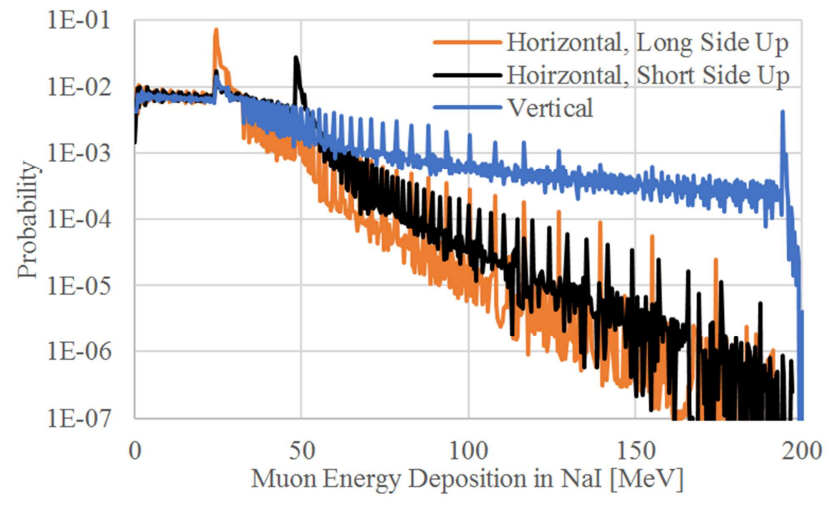

Figure 7. Energy spectra for NaI detector calculated as the product of muon's linear energy deposition and its path length distribution in the Horizontal-Long Side Up (orange), Horizontal-Short Side Up (black), and Vertical (blue) orientations.

Two other features on this spectrum are continuums. The first is a continuum of energies less than mode $\mathrm{B}$, labelled $\mathrm{A}$, which is the result of muon path lengths shorter than the detector's shortest dimension. Energies within this continuum are equally likely, and they have a probability approximately one third of that of mode B. This is largely the result of muons entering the detector through one face and exiting though an orthogonal face: entering through the top and leaving out a side, entering through a side and leaving through the bottom, or entering through one side and leaving through a perpendicular side (i.e. "cutting the corners"). The other continuum is that of energies between modes $\mathrm{B}$ and $\mathrm{D}$, labelled C. Similar to A, it is the result of muon path lengths longer than the detector's shortest dimension and shorter than its middle dimension. This continuum has a similar physical cause as that at $\mathrm{A}$, but it also is the result of muons entering and exiting parallel sides of the detector with a non-zero angle with these faces' normal vectors. Unlike A, the energies within this continuum vary in their likelihood, where the energies near B are half as likely as that of $\mathrm{B}$ and the energies near $\mathrm{D}$ are approximately one eighth as likely as B. This is a result of an energy's stronger dependence on the muon's vertical angle of trajectory that decreases in probability as the angle increases. Diagrams of the muon paths that produce continuums A and C can be seen in Figures 10 (a) and 10 (c), respectively.

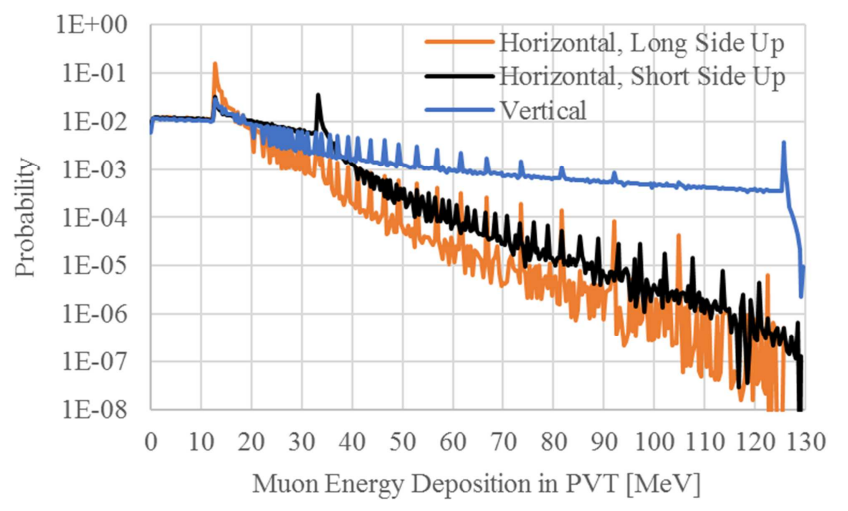

Figure 8. Energy spectra for PVT detector calculated as the product of muon's linear energy deposition and its path length distribution in the Horizontal-Long Side Up (orange), Horizontal-Short Side Up (black), and Vertical (blue) orientations.

Table 3. Comparison of measured modes'energies in the Horizontal, Short Side Up orientation with expected muon energy deposition for path lengths equal to each detector's two smallest dimensions.

\begin{tabular}{lllll}
\hline \multirow{2}{*}{ Detector } & Dimension & Calculated Muon Energy Deposition & Measured Mode Energy & Measured Mode Energy Uncertainty \\
\cline { 2 - 5 } & {$[\mathbf{c m}]$} & {$[\mathrm{MeV}]$} & {$[\mathrm{MeV}]$} & {$[\mathrm{MeV}]$} \\
\hline \multirow{2}{*}{$\mathrm{NaI}$} & 5.08 & 24.31 & 28.29 & 4.45 \\
& 10.16 & 48.62 & 48.01 & 7.55 \\
\multirow{2}{*}{ PVT } & 6.35 & 12.82 & 11.84 & 2.39 \\
& 16.51 & 33.33 & 26.09 & 5.27 \\
\hline
\end{tabular}


The last feature of the energy spectrum is for energies above that of mode D, labelled E. Energies in section E have an exponential decline in probability as energy increases up to that for the maximum muon path length, which is that of an exactly diagonal trajectory through the detector $(64.70 \mathrm{~cm}$ or $130.62 \mathrm{MeV})$. The cyclic spikes in this section are a result of both the binning scheme and the calculation resolution. These spikes can be smoothed out by either increasing the bins' width or increasing the resolution in the muons' entry locations and polar angles when doing these calculations. The latter is increasingly taxing on computer memory, but a coarser resolution causes an increasing undercount of long trajectories. Along with the exponential decline, the area near mode D has a much smaller slope than that exponential, which creates a "shoulder" on the right side of the mode at D. These are the result of muons with a variety of paths through the detector, and their energy largely depends on angle that the muon's trajectory has with the vertical axis. At lower energies, the muons enter the top of the detector with a non-zero vertical angle that is small enough for it to still leave through the bottom of the detector (i.e. path lengths slightly longer than the detector's vertical dimension). At increasing energies, the muons trajectory has an increasing vertical angle, and they

either enter through the side of the detector and leave through the bottom or enter through the top and leave out the side. At the highest energies, the muons will have a large vertical angle, resulting in a trajectory approximately parallel with the detector's longest dimension, which results in very long path lengths. This dependence on the muon's vertical angle thus produces the exponential decline in probability with energy. Examples of these possible paths can be seen in Figure 10 (e).

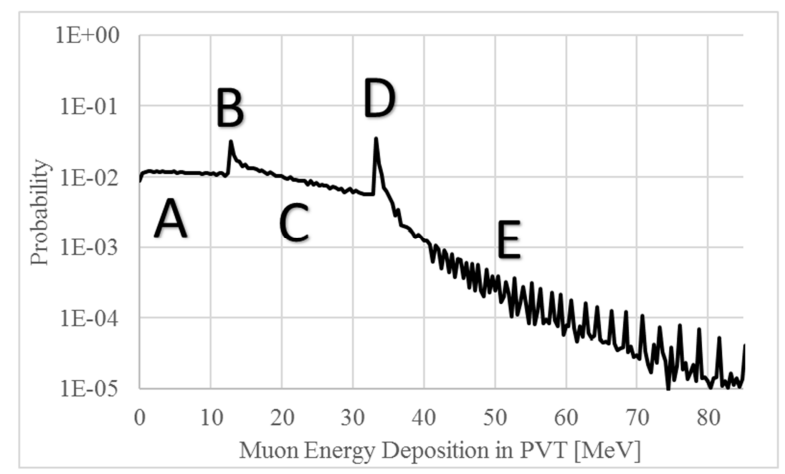

Figure 9. Calculated spectrum for the Horizontal, Short Side Up orientation of PVT labelled for various characteristics.

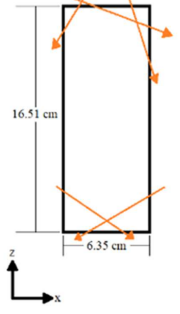

(a)

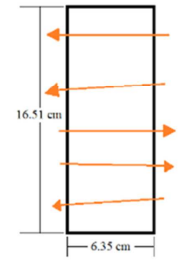

(b)

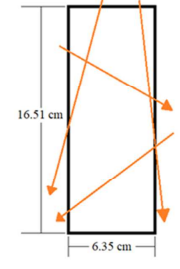

(c)

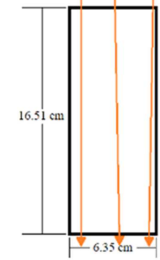

(d)

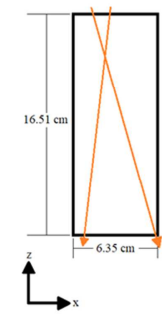

(e)
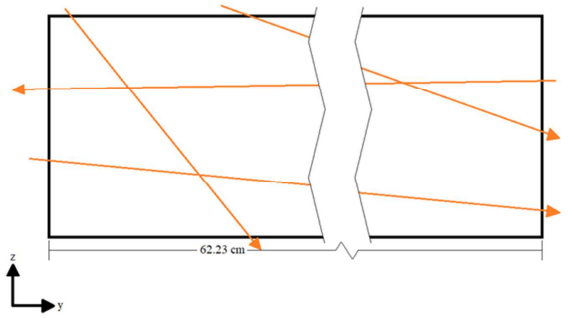

(e-side)

Figure 10. Diagrams illustrating examples of muon paths (orange arrows) through PVT in the Horizontal, Short Side Up orientation which contribute to features $A-E$, respectively labelled (a)-(e).

These features are visible with varying prominence in all three orientations for both detectors, and the energy ranges will remain the same for the same detector. For PVT in the Vertical orientation, the energy of mode B remains the same, and that of mode $\mathrm{D}$ increases to $125.6 \mathrm{MeV}$. That increase is because muons are more likely to travel vertically and thus take a $62.23 \mathrm{~cm}$ path, which means that a second mode would appear in the measured data if the detectors were calibrated to measure these higher energies. This increase naturally comes with a widening of continuum $\mathrm{C}$ (as more intermediate path lengths/energies are possible) and a contraction of section $\mathrm{E}$ (as there are only a few paths longer than the detector's longest dimension). In this case, the mode at B is more likely than that at $E$ because of the large difference between the area of the detector's side surface (which is the detector's largest surface) and that of its top surface (its smallest surface). This difference allows for 19.6 times more possible horizontal paths than vertical ones. Combining this with the difference in vertical to horizontal muon trajectories (a factor of 8.4 as stated above) suggests that mode B should be 2.3 times more likely than mode D. However, the calculated spectrum shows this difference has a factor of 7.9. This variance from expectation is likely a result of the calculation's resolution since longer paths are more likely to be undercounted with decreasing position and polar angle resolution.

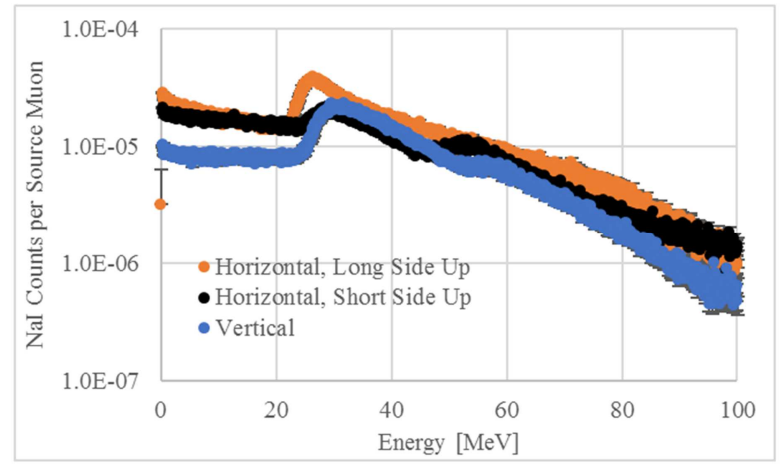

Figure 11. Simulated energy spectra for NaI detector in the Horizontal-Long Side Up (orange), Horizontal-Short Side Up (black), and Vertical (blue) orientations. 1-sigma error bars are included, but most are obscured by the data markers. 
For PVT in the Horizontal, Long Side Up orientation, the mode D disappears while the other features are still present. This, again, is the result of the difference in surface areas of the top and sides of the detector. The top is now the detector's largest surface, allowing for 1.3 times more vertical paths, which means that a muon with a vertical trajectory is 10.9 times more likely to interact with the detector than one with a horizontal trajectory. This effectively causes mode D to disappear, both in the calculated and measured spectra. Despite this aspect, the continuum $\mathrm{C}$ is still visible upon closer inspection. Like in the other horizontal orientation, that continuum appears above energies of mode B (i.e. for muon path lengths equal to the detector's shortest dimension) and below energies for muon path lengths equal to the detector's middle dimension (where mode D would be). Again, this is a result of muons (which largely enter the top of the detector) that have paths of intermediate length between the detector's shortest and middle dimensions. Besides the differences in the modes, the spectral features $\mathrm{A}$ and $\mathrm{E}$ in this orientation are largely the same as those in the other horizontal orientation.

The MCNP simulated muon spectra are shown in Figure 11 for the NaI detector and in Figure 12 for the PVT detector. The shapes of these spectra are similar to those from measurements and those from Python calculations. More importantly, similar spectral variations based on orientation are visible. The modal data for the simulations are shown in
Table 4. The energies of these modes are similar to those from measurements. This includes a prominent mode in all orientations that appears for muon path lengths equal to the detector's shortest dimension, and in the Horizontal, Short Side Up orientation, a second mode appears for path lengths equal to the detector's middle dimension. Comparing these modes to those from measured data as shown in Table 5 reveal that all the modes' energies are statistically the same at the 2- $\sigma$ level and half are the same at the $1-\sigma$ level.

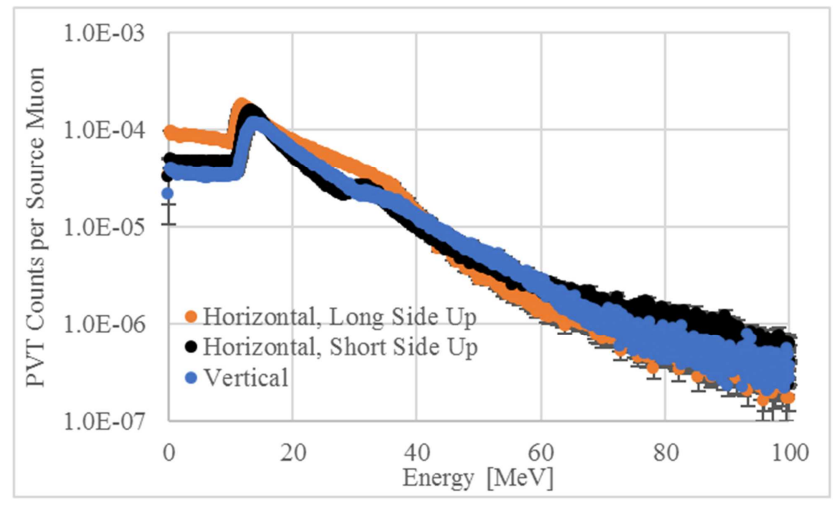

Figure 12. Simulated energy spectra for PVT detector in the Horizontal-Long Side Up (orange), Horizontal-Short Side Up (black), and Vertical (blue) orientations. 1-sigma error bars are included, but most are obscured by the data markers.

Table 4. Modal data from simulated responses of in NaI and PVT by orientation to background muons, including modes' full-widths at half maximum (FWHMs), when its calculation was possible. Missing data for "Mode 2" indicate that only one mode existed for a given spectrum.

\begin{tabular}{|c|c|c|c|c|c|c|c|c|}
\hline & & & \multicolumn{6}{|c|}{ Detector/Orientation } \\
\hline & & & \multicolumn{3}{|c|}{ NaI } & \multicolumn{3}{|l|}{ PVT } \\
\hline & & Units & $\begin{array}{l}\text { Horizontal, Long } \\
\text { Side Up }\end{array}$ & $\begin{array}{l}\text { Horizontal, } \\
\text { Short Side Up } \\
\end{array}$ & Vertical & $\begin{array}{l}\text { Horizontal, Long } \\
\text { Side Up }\end{array}$ & $\begin{array}{l}\text { Horizontal, } \\
\text { Short Side Up } \\
\end{array}$ & Vertical \\
\hline \multirow{3}{*}{ Mode 1} & Energy & {$[\mathrm{MeV}]$} & 22.316 & 28.084 & 31.824 & 11.968 & 13.396 & 13.736 \\
\hline & \multirow{2}{*}{ FWHM } & {$[\mathrm{MeV}]$} & 13.396 & $* 19.588$ & 16.796 & 7.344 & 5.984 & 8.772 \\
\hline & & {$[\%]$} & 50.9 & $* 69.7$ & 52.8 & 61.4 & 44.7 & 63.9 \\
\hline Mode 2 & Energy & {$[\mathrm{MeV}]$} & - & 52.360 & - & - & 31.960 & - \\
\hline
\end{tabular}

${ }^{*}$ Linear extrapolation used to estimate the left half-maximum value.

Table 5. Comparison of modal data for both measured and simulated background muon responses in NaI and PVT in various orientations.

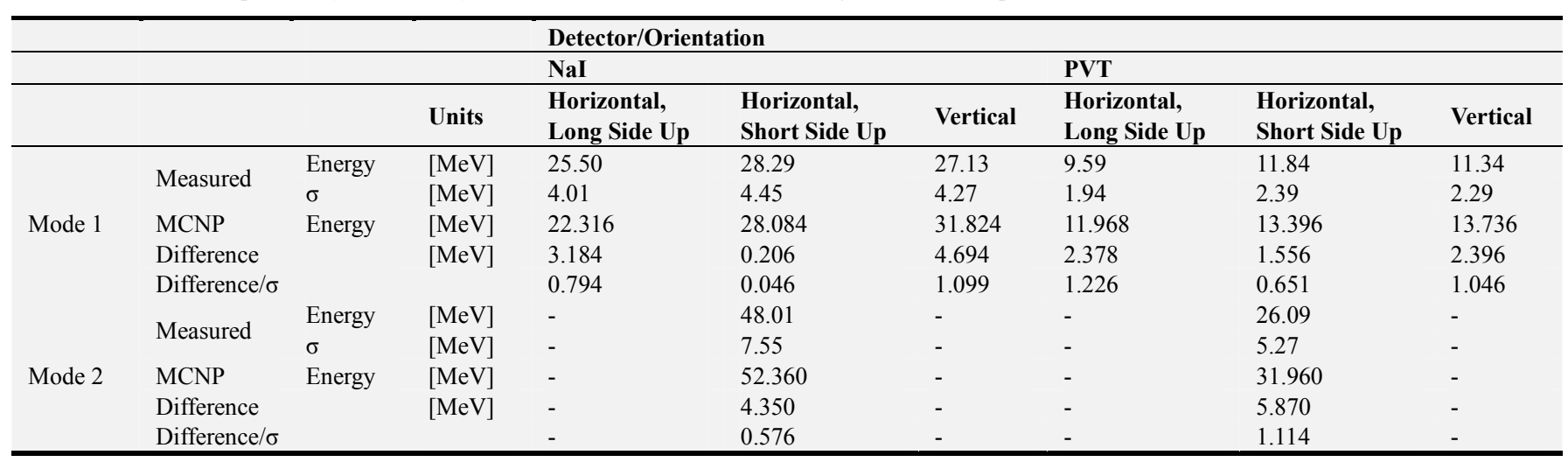

These data indicate that muons are the particle responsible for the background response in these scintillator detectors. This aspect is especially useful for mobile search systems as background muons and neutrons are largely produced by secondary cosmic ray interactions, and therefore, their fluxes would likely be proportional in most situations [13]. As mentioned above, this proportionality has been seen in operational data employing high energy scintillator measurements. These characteristics suggest that it could be possible to apply the high energy scintillator response to 
characterize background more effectively in neutron counting systems during mobile searches.

\section{Conclusion}

This work shows that the background response at high energies in scintillation detectors is mostly the result of muons. These muons produce peaks whose centroids appear at energies between $5 \mathrm{MeV}$ and $60 \mathrm{MeV}$ depending on the detector's orientation and composition. Mobile search systems commonly use these scintillation materials, and operational data (along with previous work) show that these high energy signals (caused by muons) are correlated with the background response from a neutron counter. Because of this, it seems plausible that a method could be devised to predict the background response in a neutron counting system using the background response from muons. This aspect will require further study to characterize the relationship and test the method's applicability for mobile searches.

\section{References}

[1] G. F. Knoll, Radiation Detection and Measurement, 4th Edition, John Wiley \& Sons, 2010.

[2] T. Ichimiya, T. Narita, K. Kitao, "Natural background gamma-ray spectrum List of gamma-rays ordered in energy from natural radionuclides (JAERI-Data/Code--98-008)," Japan Atomic Energy Research Institute, 1998. https://inis.iaea.org/collection/NCLCollectionStore/ Public/29 /033/29033666.pdf.

[3] S. Hayakawa, Cosmic Ray Physics - Nuclear and Astrophysical Aspects, John Wiley \& Sons, 1969.

[4] J. N. Wagner, C. Marianno, T. McCullough, "Parking Garage Measurements Indicating a Gamma Spectrometer-Neutron Counter Background Correlation," International Journal of Nuclear Security, Vol. 6: No. 1, Article 6. 2020. Available at: https://trace.tennessee.edu/ijns/vol6/iss1/6/.

[5] Alpha Spectra, Inc. "ASI 2" x 4" x 16" Detector Data Sheet," available

https://alphaspectra.com/wp-content/uploads/ASI-2in-x-4in-x16in-NaITl-Data-Sheet.pdf (accessed on 7 September 2020).
[6] Alpha Spectra, Inc. "Nomenclature - ASI Model Numbers Explained," available at https://alphaspectra.com/nomenclature/ (accessed on 7 September 2020).

[7] Alpha Spectra, Inc. "ASI-100 Plastic Scintillator," available at https://alphaspectra.com/wp-content/uploads/ASI-100-PlasticScintillator.pdf accessed on 7 September 2020).

[8] AMETEK Inc. - ORTEC, "digiBASE 14-Pin PNT Tube Base with Integrated Bias Supply, Preamplifier, and MCA (with Digital Signal Processing) for NaI Spectroscopy," available at https://www.ortec-online.com/-/media/ametekortec/brochures/ digibase.pdf (accessed on September 7th, 2020).

[9] AMETEK Inc. - ORTEC, "MAESTRO Multichannel Analyzer Emulation Software," available at https://www.ortec-online.com/-/media/ametekortec/brochures/ maestro.pdf?dmc $=1 \&$ la $=$ en accessed on September 7th, 2020).

[10] Gooding, T. J. and Pugh, H. G. "The Response of Plastic Scintillators to High-energy Particles" Nuclear Instruments and Methods. Vol. 7 (1960) pp. 189-192.

[11] Taylor, C. J., et. al. "Response of Some Scintillation Crystals to Charged Particles." Physical Review. Vol. 84, No. 5 (1 December 1951). pp. 1034-1043.

[12] W. Preusse and S. Unterricker, "The contribution of cosmic ray muons to the background spectrum of gamma ray spectrometers." Nuclear Instruments and Methods in Physics Research B. Vol. 94 (1994) pp. 569-574.

[13] J. F. Ziegler, "Terrestrial Cosmic Rays", IBM Journal of Research and Development, 1996, p 23.

[14] P. K. F. Grieder, Cosmic Rays at Earth - Researcher's Reference Manual and Data Book, Elsevier, 2001.

[15] C. Y. E. Ho, "Cosmic Ray Muon Detection Using NaI Detectors and Plastic Scintillators," University of Virginia. Available https://home.fnal.gov/ group/WORK/muonDetection.pdf accessed on September 7th, 2020).

[16] D. E. Groom et. al, "Muon Stopping Power and Range Tables $10 \mathrm{MeV}-100 \mathrm{TeV}$," Atomic Data and Nuclear Data Tables, Vol 76, No. 2. July 2001.

[17] C. J. Werner, et al., "MCNP6.2 Release Notes", Los Alamos National Laboratory, report LA-UR-18-20808 (2018). 